\title{
FedEx and UPS Network Structure and Accessibility Analysis Based on Complex Network Theory
}

\author{
Changsong Zhao $\mathbb{D}^{1},{ }^{1}$ Chunliang Xiu $\mathbb{D}^{2},{ }^{2}$ and Guanyi Yu ${ }^{1}$ \\ ${ }^{1}$ School of Business Administration, Northeastern University, Shenyang 110169, Liaoning, China \\ ${ }^{2} J a n g H o$, Architecture College, Northeastern University, Shenyang 110169, Liaoning, China \\ Correspondence should be addressed to Chunliang Xiu; xiuchunliang@mail.neu.edu.cn
}

Received 17 October 2020; Revised 5 January 2021; Accepted 12 January 2021; Published 27 January 2021

Academic Editor: Anirban Chakraborti

Copyright (c) 2021 Changsong Zhao et al. This is an open access article distributed under the Creative Commons Attribution License, which permits unrestricted use, distribution, and reproduction in any medium, provided the original work is properly cited.

\begin{abstract}
With the expansion of the global air cargo transport system, the operating structure of air cargo has become increasingly separate from passenger counterpart, forming an independent organization model. Despite the Chinese air cargo capacity has grown exponentially in the past, its network is still in its infancy. FedEx and UPS have well-established air cargo networks and have operated effectively on both international and domestic scale; thus, understanding the structure and evolution of their air cargo networks is of a high reference value. In conjunction with the division of US regions from the United States Geological Survey (USGS), this paper refers to FedEx and UPS as FEPS and analyzes its topological structure, complexity properties, and air cargo accessibility by using social network analysis (SNA) and accessibility evaluation methods. The results suggest that (1) the structure of the FEPS air cargo network is in the highly developed states and has the typical "small-world" and "scale-free" network characteristics; (2) the degree centrality values for the nodes in the FEPS network suggest that the network complexity has increased; (3) airports in Memphis (MEM), Louisville (SDF), Indianapolis (IDN), and Ontario (ONT) are the major hubs with both high centrality values and air cargo accessibility; and (4) the FEPS network presents a unique hub-and-spoke structure compared with the passenger counterpart.
\end{abstract}

\section{Introduction}

Air transport has a pivotal role in global and regional economic development. Air cargo transfers 35\% of international trade value through only $1 \%$ of the total trade volume [1]. Additionally, with the booming of e-commerce, major air cargo carriers such as Amazon, Jingdong, and Alibaba rely heavily on the express delivery services, leading to an increase in air transport infrastructure development.

Additionally, flying air cargo naturally involves cargo handling, ground transportation, and different routing strategies, which are not present in passenger networks, and therefore, it is of high reference value to study the unique properties of the hub-and-spoke air cargo network and their rationale for the formation. For air cargo carriers operating on a similar scale, such as Chinese air cargo carriers Shunfeng Express and Yuantong Express who are still planning their dedicated airline routes for their air cargo transport network, comprehending the network property provides significant valuable insights for their network design.

Regardless of its importance, the air cargo industry is not regarded as important as the air passenger counterpart. Additionally, a tremendous amount of case studies and literature focuses on the structural characteristics of air passenger transport over the past decades, and yet, for a long time, the air cargo industry has existed as an adjunct to passenger transport. The rapid growth of the air cargo industry leads to the increasing structural separation of air passenger and cargo network, and the differences between networks are becoming increasingly evident. Air cargo hubs and route organizations display different network characteristics and properties from passenger transport. The U.S. air cargo development has been leading the world, with 
FedEx and UPS are the two most important air cargo integrators together accounting for more than $75 \%$ of the U.S. air cargo market in 2018 (as shown in Table 1). Studying the air cargo network jointly formed by the two companies can clarify the basic characteristics of the U.S. air cargo network pattern and discover the unique features of the cargo network that differ from air passenger transportation, which is of academic significance, and can provide an empirical reference for other countries in building new air cargo networks with application value.

Our contribution is (i) to fill the gap in the literature of comprehending the air cargo network structure from an enterprise perspective, (ii) to analyze the complex network properties and the correlation between centrality measures, and (iii) to provide an index to measure air cargo accessibility from a complex network theory perspective. The rest of the paper is structured as follows. Section 2 summarizes the literature concerning air cargo transportation from a geological economic perspective. Section 3 briefly describes the history of FedEx and UPS, also the data used in this research. Section 4 and 5 focus on the complex network measures and air cargo accessibility index. Section 6 concludes this study and discusses some further research possibilities.

\section{Literature Review}

The existing literature on the air cargo network addresses their geographic characteristics, accessibility, robustness, and the economic benefits of the air cargo network structure. From an economic development perspective, the authors of [2] suggested that the air transport network fosters economic growth in China and the Chinese air transport network centered in Beijing, Shanghai, Guangzhou, and Shenzhen, covering the Pearl River Delta (PRD), Yangtze River Delta (YRD), Bohai Sea Economic Zone, and ChengduChongqing Economic Zones. The authors of [3-6] discovered that there are overlaps between the Chinese air passenger network structure and economic development zones by analyzing the Chinese air passenger enterprises. The authors of [7] used gravity models to access the Chinese air cargo network and concluded that the Chinese air cargo market is still emerging and is still depends heavily upon passenger networks. The authors of [8] further analyzed the Chinese air cargo network and identified the international trade drivers using the augmented gravity model, considering cultural influence as socio-economic factors that affect the cargo demand. The authors also concluded that the domestic air-freight network in China is primarily composed of point-to-point, and the leading airports are yet to become cargo gateway hubs, and the composition of the economy is more important than the size of the economy. The authors of [9-11] compared the different air transport network structures and concluded that the structure of the air passenger networks is subject to the local socio-economic factors. The authors of [12] put forward the idea that air transport hubs have become the engine of the economy and conceptualized a new urban form centered on airports, namely, Aerotropolis. The authors of [13] confirmed that air transport infrastructure boosts the local economy.
Scholars have extensively depicted the structure of the air transport network using complex network theory and graph theory. The authors of $[14,15]$ provided a preliminary analysis of the world-wide airline network (WAN) through complex network theory and proposed a new model with geopolitical constraints. The author of [16] described the FedEx and UPS networks by their numbers of nodes and edges in an undirected network. The authors of [17] analyzed the city of Harbin and proposed to re-evaluate the unique geographic value of gateway airports. The authors of $[18,19]$ explored weighted networks using the example of the airport network (WAN) and the scientist collaboration network (SCN). The authors of [20] discovered the travel time between airport pairs in the European air passenger network, while the authors of [21] discussed the multilayer structure of the European ATN. The authors of $[22,23]$ analyzed the Chinese air transport network and its multilayered features. The authors of [24] suggested a strong relationship between air cargo and trade volume, noting that the oil price has a critical impact on the air cargo network. Malighetti et al. [25] discovered that FedEx, UPS, and DHL operated extensively through a multihub structure, and all of them focus on the flexibility and robustness of their networks. The authors of [26] analyzed the global air transport network and concluded that the air cargo network has a different structure than the passenger network, and bridging airports are more valuable.

Hansen [27] defined accessibility as the convenience of reaching a destination from a given location. In the past, the study of airport accessibility primarily focuses on reachability from either land or air location. Additionally, the competitiveness of passenger airports is severely affected by service quality [28]. The authors of [4] investigated the Chinese air passenger network accessibility from 2001 to 2013 and discovered that Beijing, Shanghai, Guangzhou, and Shenzhen have high passenger accessibility. Meanwhile, airport air-side accessibility increased in the southwest region, whereas the northeast gradually decreased. However, research on cargo accessibility is scarce despite the value created by the air cargo sector.

Other studies focus on the robustness of the air transport network using a variety of attacking strategies on the air transport network [29-32] though none of these studies discussed the implications for air cargo enterprises and their network structures.

\section{Scope of the Study}

Airports have become important nodes in the transportation economy, and the structure of the air cargo network not only reflects the connections between the local economy but also affects regional economic development. In recent years, the Chinese aviation industry has grown exponentially in terms of both size of the network and the cargo volume. Airports such as PEK, PVG, HKG, and CAN in Beijing, Shanghai, Hong Kong, and Guangzhou have already surpassed the top U.S. air cargo hubs such as MEM and SDF in Memphis and Louisville. However, the Chinese air cargo network still operates under the framework of its passenger counterpart 
TABLE 1: FEPS domestic market share 2008-2018.

\begin{tabular}{lccc}
\hline Year & FedEx market share (\%) & UPS market share (\%) & FEPS market share (\%) \\
\hline 2008 & 47.69 & 30.24 & 77.93 \\
2009 & 53.91 & 29.80 & 83.71 \\
2010 & 54.23 & 29.34 & 83.57 \\
2011 & 54.78 & 29.82 & 84.60 \\
2012 & 55.43 & 29.57 & 85.00 \\
2013 & 55.86 & 29.43 & 85.29 \\
2014 & 54.84 & 30.01 & 84.85 \\
2015 & 54.08 & 29.07 & 83.15 \\
2016 & 52.58 & 28.63 & 81.21 \\
2017 & 49.44 & 28.29 & 77.73 \\
2018 & 48.98 & 26.79 & 75.77 \\
\hline
\end{tabular}

and soon would be challenged by the increasing international trade and domestic market demand. Given the long history and depth of involvement of FedEx and UPS, the US Department of Transportation (US DOT) distinguishes FedEx and UPS as the only two air cargo carriers in GROUP III, which have operating revenue over a billion USD, and therefore, it is meaningful to study their network topological structure, complex network characteristics, and cargo accessibility.

Air cargo network has become an increasingly important part of the transportation economy, and its structure reflects not only the connectivity of the local economy but also serves as an indicator for regional economic development. In recent years, the Chinese aviation industry has grown exponentially in both size of the network and the cargo volume.

3.1. Brief History of FedEx and UPS. Founded in 1965, FedEx moved its headquarters from Little Rock, Arkansas, to Memphis, Tennessee, in 1973. FedEx was founded on the idea of creating an efficient air transport system for timesensitive goods such as pharmaceuticals and chipsets, independent of the passenger network. In the late 1970s and early 1980s, FedEx actively participated in aviation policy reforms, expanding its operating area to Canada and establishing its aviation hub base at Memphis International Airport. At the beginning of the 21st century, FedEx created a multimodal transport network covering multiple regions of the world and logistics-related industrial chains through the acquisition of many logistics companies in other countries and regions.

United Parcel Service (UPS) was established in Seattle in 1907. In 1909, it cooperated with the U.S. Postal Service. In the 1930s, UPS was able to provide transportation services that covered the entire West Coast of the United States, becoming the first U.S. carrier to offer air cargo services. During World War II, the company expanded to the East Coast. In the 1950s, UPS began to build private roads, combined with its ground transportation, and full-air-freight-service was introduced. With the relaxation of U.S. aviation policy in the 1980s, UPS began to develop its fleet and obtained permission from the Federal Aviation Administration to set up UPS Aviation. In 1989, UPS expanded its business overseas for the first time and expanded to the rest of the world, forming the global air cargo network landscape.

3.2. Data Selecting and Processing. The data for this study consist of eleven years of air cargo and freight data from the US DOT Bureau of Transportation Statistics (BTS) T-100 market data, which contains the OD pair of airports with cargo and passenger volume and flight distance between origin and destination. This study focuses on the domestic air cargo network, and therefore, international routes are excluded.

(1) Considering that the production and transport of goods are closely related to the development of the local economy, we consider the FEPS network as a directed graph. We break down the stopover flights $\mathrm{A}-\mathrm{B}-\mathrm{C}$ to $\mathrm{A}-\mathrm{B}$ and $\mathrm{B}-\mathrm{C}$, and the direction of the flights is distinguished.

(2) The O-D network is built with the airport as a node and route as edges, and the cargo volume Freight Ton Kilometers (FTK) of the airport nodes is used as a weight to analyze the topological structure, complex network properties, and cargo accessibility.

(3) The United States Geological Survey divides the United States territory into the Pacific, Rocky Mountains, Southwest, Northeast, Southeast, Midwest, Alaska, and Hawaii. We describe the topology in conjunction with the USGS division.

\section{Methodology}

\subsection{Complex Network Indices}

4.1.1. Degree and Degree Distribution. One of the key properties of nodes in the network is the degree of a node. The degree $k_{i}$ of a node refers to the number of links it has to other nodes. The average degree of all nodes in the network becomes the average degree of the network, denoted as $k$. The degree distribution of nodes in the network is represented by the probability distribution function $p(k)$ and the cumulative probability distribution function $P(k)$. When $p(k)$ and $P(k)$ are power functions, the structure is said to have a "scale-free" property expressed as follows: 


$$
P(k)=\sum_{k_{\prime}=k}^{\infty} p(k) .
$$

Previous studies have shown that $p(k)$ of a random network has a similar Poisson distribution. Many studies have attributed the topology of real networks to conform to power-law distribution characteristics $P(k) \propto k^{-\lambda}(2 \leq \lambda \leq 3)$ of "scale-free" network [33].

4.1.2. Average Path Length $(L)$. The average path length $L$ of the network is defined as the average distance between any two nodes, which can reflect the strength of network accessibility, and it can be used to present the mean distance of any two nodes of a network written as follows:

$$
L=\frac{1}{n(n-1)} \sum_{i \neq j} d\left(v_{i}, v_{j}\right)
$$

4.1.3. Cluster Coefficient $\left(C_{i}\right)$. Clustering coefficient measures how closely a node is connected to its neighbor, defining as the ratio of the connected edges $E_{i}$ with all its neighbors to the maximum number of possible links. The clustering coefficient $(C)$ is the mean value of all the single nodes, as the ratio of the number of edges between a node and the maximum number of edges may be connected to all nodes connected to it. For nodes, $v_{i}$, its adjacent node collection, the number of actual variables in $N_{i}$ is as follows:

$$
E_{i}=\frac{1}{N} \sum_{v_{i} \in V} e_{j k}
$$

For nodes in a directed network, the clustering coefficient of $v_{i}$ is expressed as

$$
C_{i}=\frac{E_{i}}{k_{i}\left(k_{i}-1\right)} .
$$

The higher the $C_{i}$ is, the closer the nodes are connected. Average clustering coefficient $C$ is defined as the average of all node cluster coefficients. High $C$ indicates stronger connections within the network.

$$
C=\frac{1}{n} \sum_{i=1} C_{i}
$$

4.1.4. Degree Centrality (DC). Degree centrality is measured by the degree of the given airport in the network. It reflects the size of the node's direct contact with other nodes in the network. The node centrality indicates the likelihood of contact with other nodes, the larger the value, the greater probability of connections with other nodes, and it is defined as follows:

$$
D C_{i}=\frac{1}{n-1} k_{i}
$$

4.1.5. Closeness Centrality (CC). Closeness centrality is measured by the sum of the shortest distances from a given node to all nodes, reflecting the relative accessibility of the node in the network.

$$
C C_{i}=\left[\frac{1}{n-1} \sum_{j=1, j \neq 1} d_{i j}\right]^{-1} .
$$

4.1.6. Betweenness Centrality (BC). The betweenness centrality is measured with the number of shortest paths, between any couple of nodes in the graph that passes through the target node, reflecting the node's transit and bridging capabilities in the network.

$$
B C_{k}=\frac{2}{n^{2}-3 n+2} \sum_{i=1, j \neq k}^{n} \sum_{j \neq k}^{n} \frac{\delta_{i j}^{k}}{\delta_{i j}}
$$

where $\delta_{i j}$ represents the total number of shortest paths between node $v_{i}$ to $v_{j}$. The larger the $B C_{k}$ is, the stronger the hub-and-spoke effect of the network has.

4.2. Air Cargo Accessibility (ACA) Index. Referring to the Reynolds [34] method of measuring passenger accessibility from Ireland to other airports in Europe by the number of seats between routes, air cargo accessibility in this study is measured by the weighting of freight and centrality between airports as follows:

$$
A i=\sum_{i=1}^{n} F T K_{i j} \times W_{D C_{i}}, \quad i=1,2,3, \ldots, n,
$$

where $A_{i}$ represents airport cargo accessibility, $n$ for the total number of airports, $A F T_{i j}$ (Freight Ton Kilometer) represents the air cargo volume from airport $i$ to $j$, and $W_{D C}$ represents the sum of in-degree and out-degree of airport $j$; equation (9) indicates that the $A_{i}$ airport's accessibility is equal to the weighted sum of the annual cargo volume and connectivity of the airport's arrival at the other airports.

$$
A I_{i}=\frac{A_{i}}{(1 / n) \sum_{i=1}^{n} A_{i}} \quad i=1,2,3, \ldots, n .
$$

According to equation (10), the accessibility of FEPS airports from 2008 to 2018 is calculated, and the change of airport node canary is analyzed according to the average of the reachability, the standard deviation, and other statistical parameters. The ratio of airport $i$ to the average reachability $A I_{i}>1$ indicates that airport $i$ has accessibility above or equal to the average accessibility of all airports.

\section{Findings}

5.1. FEPS Network Topology Characteristics. As seen in Figure 1, airports in the Midwest and Southeast regions have better connectivity. We divided the airports in the FEPS network into five hierarchical groups based on their cargo volume. The size of the FEPS network is reflected by the number of nodes and edges, which has not drastically changed from 2008 to 2018. 
Between 2008 and 2014, US domestic air cargo volume increased as the US economy (and the world) recovered from the global financial crisis. However, the air cargo growth rate slowed between 2015 and 2018. The number of FedEx airports increases as FedEx finished acquiring TNT in 2016. In 2017, both FedEx and UPS shifted their focus to multimodal transportation by closing their nonhub, low cargo capacity airports. Meanwhile, the complexity of the network has increased. By the end of 2018 , the average degree in the network increased by $6 \%$ from 8.374 in 2008 to 8.925 in 2018, which indicates stronger network connectivity (as seen in Table 2). Meanwhile, the average clustering coefficient increased from 0.419 to 0.572 , indicating connections amongst nodes are stronger. 281 FEPS airports scattered across the country with 62 airports in the Midwest, 54 in the Southeast, 53 in the Pacific, 33 in the Northeast, 29 in the Rocky Mountains, 27 in Southeast, and 23 in noncontiguous regions. However, the hub establishment of the FEPS network is not aligned with the fast economic development regions. According to the US Bureau of Economic Analysis, major economic growth took place in a part of the Rocky Mountain, Pacific, and Southeast region, where the FEPS hubs are not located, indicating the different requirement for the hub establishment for the air cargo network.

Compared with the passenger hub locations such as Chicago, New York, Atlanta, and San Francisco, air cargo hub selection considers neither the needs for round trip flights nor the convenience to go more tourist destination. Instead, air cargo hubs focus more on places with higher geographic accessibility. For instance, MEM and SDF in Memphis and Louisville have flights that can reach any city in the nation within 4 hours.

5.2. Complex Network Properties. The probability of the cumulative degree of the FEPS network in 2008, 2011, 2016, and 2018 is calculated (as shown in Figure 2). All of which follows a power-law distribution with a long tail on the right, $R^{2}>0.85$, indicating that a large number of nodes are connected through a small number of hubs via multiple edges, portraying a typical hub-and-spoke network structure, where most of the nodes are connected through a few hub nodes.

Figure 3 displays the geographic distribution of nodes centrality values. The overall centrality values (DC, CC, and $\mathrm{BC}$ ) have increased, specifically, DC and CC increased in the Rocky Mountains and Southwest regions; however, there is no significant increase in the $\mathrm{BC}$, indicating that the nodes with strong transshipment capability have not increased drastically, while an increasing number of nodes have more direct connections.

Tables 3-6 summarize the change in the rankings of complex measures, which reflects the complexity of the network and its structural characteristics, especially the hub nodes. The change of the centrality values suggests the routing strategies in the FEPS network are constantly adjusting over time, resulting in shifts of complexity in the network. Only MEM and SDF ranked highest by all three indices, which implies these two nodes as the core hubs. Other airports have rather volatile changes in their centrality measures. For instance, Philadelphia (PHL) in 2008 (Table 3) has a degree of 100 (ranked $8^{\text {th }}$ ) and dropped to 84 in 2011 (Table 4) and further 82 in 2016 (Table 5) and eventually raised to 89 (Table 6) in 2018 (ranked $6^{\text {th }}$ ), and the closeness centrality (CC) dropped from 0.459 (ranked $4^{\text {th }}$ ) to 0.448 (ranked $13^{\text {th }}$ ).

This suggests that there is no trend in the alteration of the centrality measures for any single node in the network, and more importantly, some nodes gained more transit capability or ground handling support, which might have moved up in the rankings and serve as the local or regional warehouse. Interestingly, the gateway airports such as ATL, LAX SEA, and ORD do not exhibit strong rankings in all three centrality values; though those airports serve as international mega-hubs for passenger network, it has a very limited impact on the air cargo network, which further suggests separation of the cargo and passenger network structure and their unique structural properties.

Figure 4 further illustrates the relations between degree centrality (DC), betweenness centrality (BC), and closeness centrality (CC). As seen in Figure 4, the correlation between $\mathrm{DC}$ and $\mathrm{BC}$ increased from 0.859 to 0.871 , and the correlation between DC and CC decreased from 0.714 to 0.612 while the correlation between CC and BC dropped from 0.836 to 0.527 .

This indicates three scenarios regarding the network complexity properties.

(1) The probability of nodes having numerous direct connections (DC) and having high transit capability (BC) increased, meaning that the hub airports in the network have stronger transshipment capability at the same time they have more directions to other nodes in the network, indicating hub-and-spoke effects increase.

(2) The probability of nodes having both numerous direct connections (DC) and a large sum of shortest paths (CC) from other nodes reduces. In other words, many nodes are becoming terminal nodes, in which case, increase the total number of directions; at the same time, these nodes do not serve as the transit nodes.

(3) The probability of nodes has both a large sum of shortest paths (CC), and strong capability of transit (BC) decreased, meaning that the bridging nodes in the networks are distancing from other smaller nodes. 


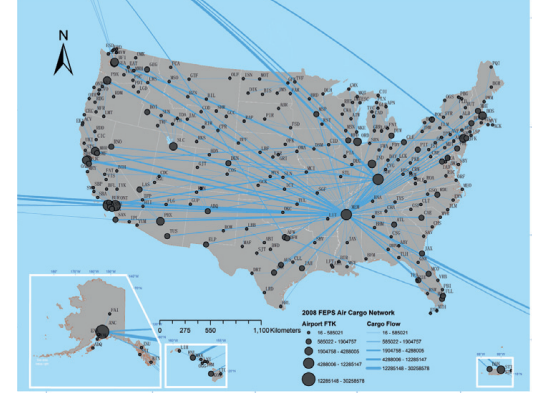

(a)

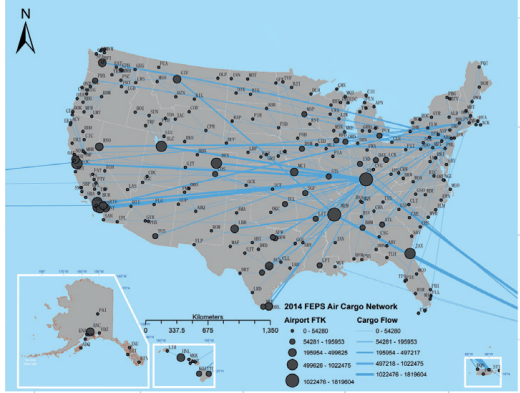

(b)

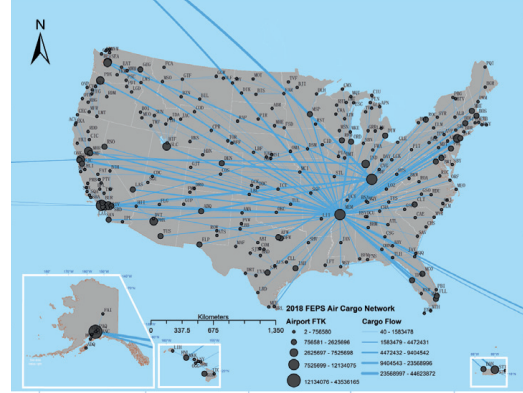

(c)

FIGURE 1: FEPS air cargo network topology in 2008, 2014, and 2018.

TABLE 2: FEPS network complex network characteristics from 2008 to 2018.

\begin{tabular}{lcccccccc}
\hline \multicolumn{2}{l}{ FEPS air cargo network characteristics } & \multicolumn{2}{c}{ Rerage clustering } \\
Year & Nodes & Routes & Diameter & $\begin{array}{c}\text { Average } \\
\text { degree }\end{array}$ & $\begin{array}{c}\text { Average path } \\
\text { length }\end{array}$ & $\begin{array}{c}\text { Average path } \\
\text { length }\left(L_{\text {rand }}\right)\end{array}$ & Clustering coefficient $\left(C_{\text {rand }}\right)$ \\
\hline 2008 & 280 & 2221 & 6 & 8.347 & 0.419 & 2.93 & 2.62 & 0.031 \\
2011 & 284 & 2401 & 6 & 8.458 & 0.505 & 2.954 & 2.672 & 0.031 \\
2015 & 279 & 2522 & 6 & 9.362 & 0.562 & 2.422 & 2.372 & 0.045 \\
2016 & 280 & 2540 & 6 & 8.411 & 0.576 & 2.981 & 2.774 & 0.028 \\
2018 & 281 & 2508 & 6 & 8.925 & 0.572 & 2.931 & 2.566 & 0.032 \\
\hline
\end{tabular}

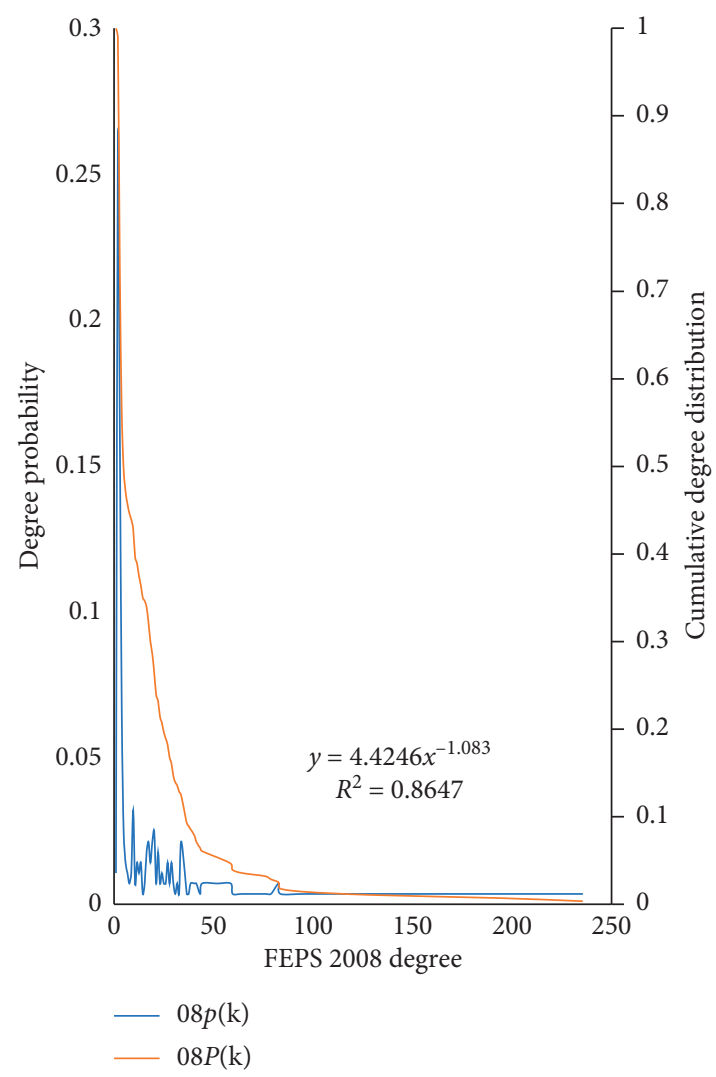

(a)

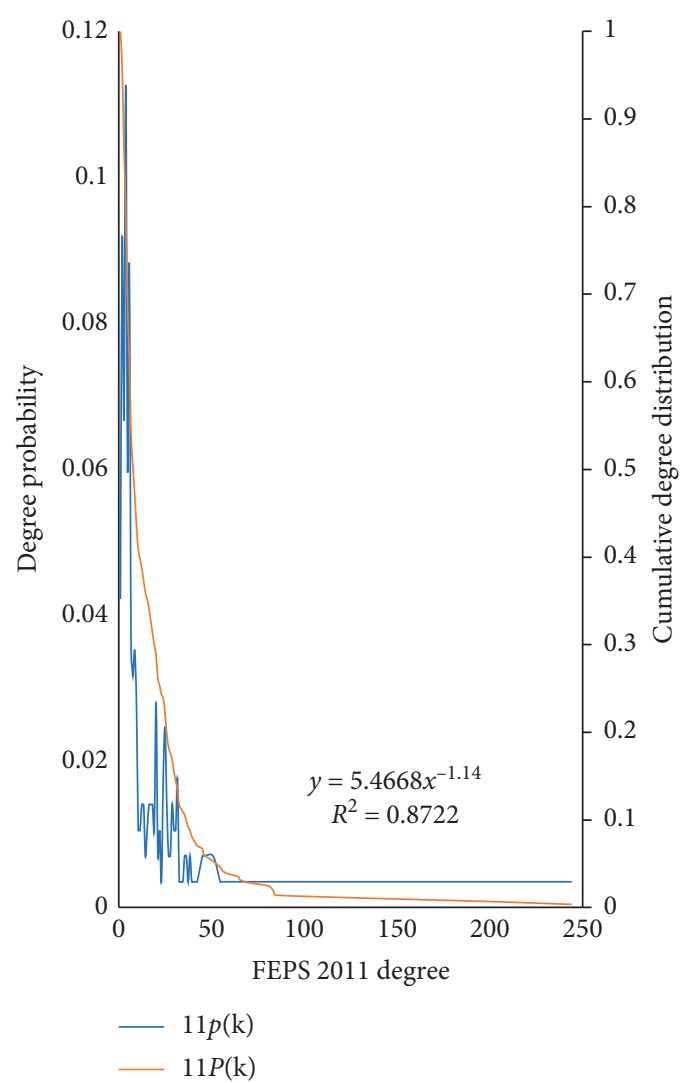

(b) 


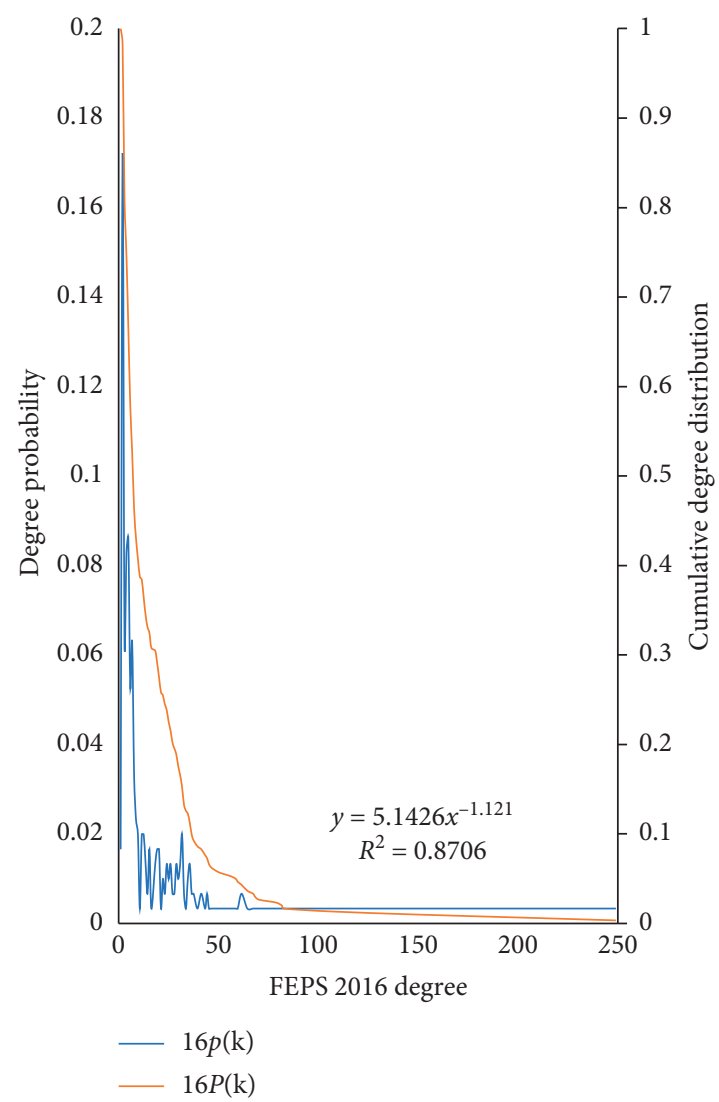

(c)

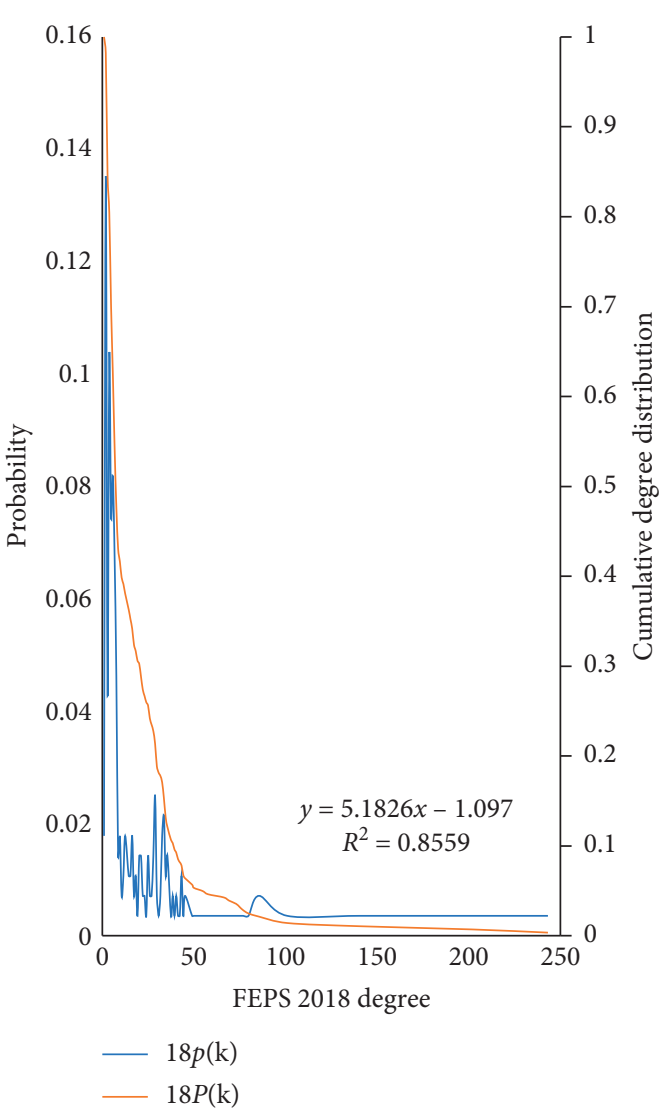

(d)

Figure 2: Cumulative degree distribution of the FEPS network in 2008, 2011, 2016, and 2018.

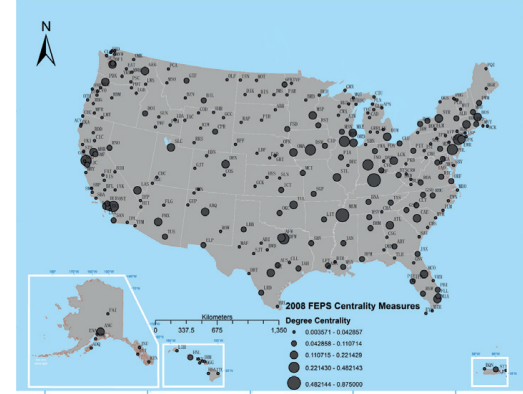

(a)

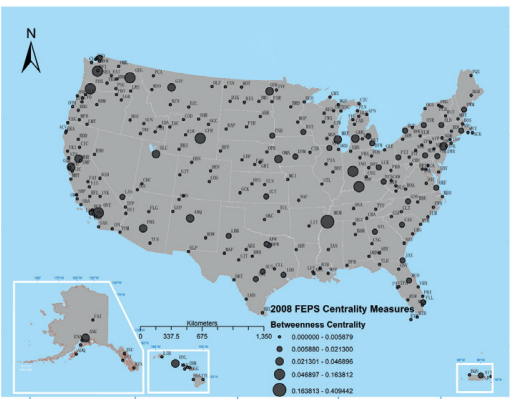

(c)

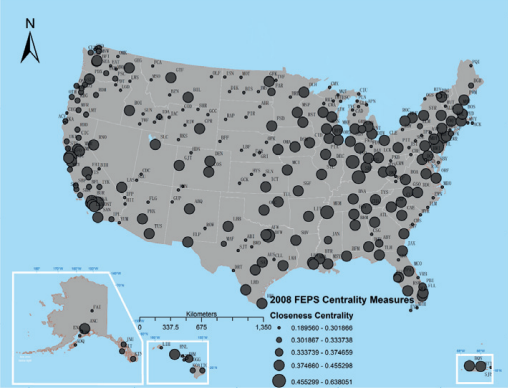

(b)

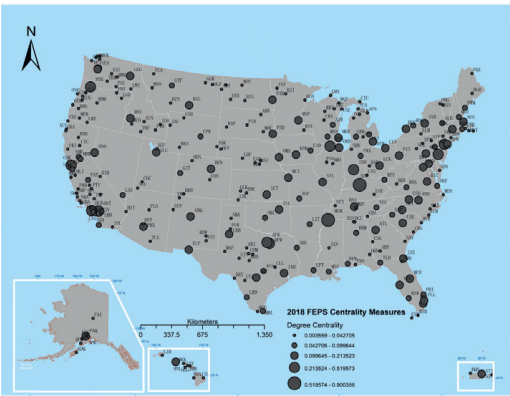

(d) 


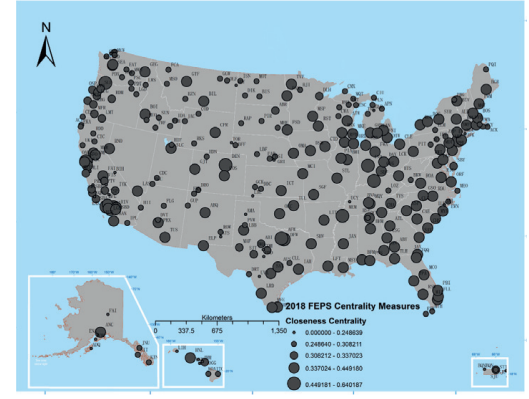

(e)

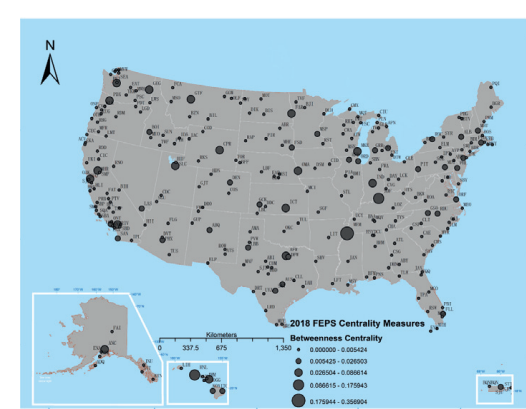

(f)

FIGURE 3: Spatial distribution of degree centrality, closeness centrality, and betweenness centrality of FEPS air cargo network in 2008 and 2018.

These properties suggest that many existing bridging hubs are evolving into major hubs, which have long distances to the remote nodes. Additionally, the number of nodes with degree value less and equal to 2 increased from 38 (13\% of total nodes) to 44 (15\% of total nodes), indicating that there are more nodes in the network emerging as the terminal destination. Also, the proportion of airports with zero betweenness centrality reduced from $45.5 \%$ to $33 \%$, indicating an increase in the number of relay airports within the FEPS network. According to the division of USGS, the airport hubs mainly concentrated in the southeastern, followed by the Midwest Region, Pacific, Northeast and Southwest, and the Rocky Mountains regions.

5.3. The FEPS Air Cargo Accessibility Index (ACA). The success of the economy depends on the well-performing air cargo transportation system; measuring approaches are needed to access and track this performance in terms of the system's ability to provide access to the market. This paper employs the standard deviation coefficient to measure the change in air cargo accessibility. The results indicate that the average air cargo accessibility of FEPS airports increased by $27 \%$ in 2008-2018. During 2008-2014, the accessibility grows from 38.52 (million ton-kilometers) to 44.22 (million ton-kilometers), an increase of $14 \%$. Growth rates slowed from 2015 to 2018, with the average accessibility rate increasing by $11 \%$. Cargo accessibility increased to 49.2 (million ton-kilometers) in 2018. The number of airports in FEPS with accessibility coefficients greater than 1 increases from 20 to 30 during 2008-2018, increasing from $7 \%$ to $10 \%$, which indicates that the majority of airports have below the average accessibility. The number of airports with cargo accessibility coefficients greater than 1 in the FEPS network increased from 20 to 30 in 2008-2018, increased from $7 \%$ to $10 \%$, indicating that $90 \%$ of airports in the network have below-average air cargo accessibility, and therefore only a few airports in the FEPS network serve as hub airports. The air cargo accessibility standard deviation increased by $22 \%$ and the standard deviation coefficient decreased by $4 \%$, indicating an increase in the absolute difference in accessibility between airports but decreased as the average airport accessibility increased. The number of airports with more than 1 million tonkilometers increased from 114 in 2008 to 119 in 2018, increased by $4 \%$. Most of the hub airports are in mid-size cities, with high GPD growth; also, these hub airports have both strong demand and transit capability. The characteristics of FEPS air network airport cargo accessibility are as follows (see Table 7):

(1) The high cargo accessibility of the hub airports represented by MEM, SDF, ANC, ONT, IND, OAK, EWR, and LAX indicates that these airports have a significant position as hubs in the network. From a regional perspective, FEPS airport cargo accesses are higher in the Southeast, Midwest, and Pacific and lower in the Rocky Mountains, Southwest, and Southeast.

(2) FEPS airport cargo accessibility and its growth rate are not proportional. The top 20 airports in FEPS experienced negative annual average cargo accessibility, including SDF, ORD, BOS, SEA, DFW, and SLC, with negative growth rates of $-4.01 \%,-8.3 \%,-9.83 \%,-11.9 \%,-26.91 \%$, $-39.75 \%$, and $-42.71 \%$. The negative growth in SDF, however, does not affect the hub and topranking status of the FEPS hub. O'Hare, DFW, SEA, and BOS are the main passenger hub airports, which have reduced cargo accessibility, indicating a growing gap between FEPS air cargo networks and passenger networks. However, Los Angeles, as a larger passenger hub airport, had the highest growth rate of $137.78 \%$, followed by Phoenix 95.04\%, Portland 84.04\%, Fort Worth $60.78 \%$, Oakland 59.18\%, Salt Lake City 55.34\%, Memphis $44.13 \%$, Indianapolis $35.32 \%$, Ontario $31.01 \%$, Anchorage 25.9\%, Newark $18.37 \%$, and Philadelphia 13.44\%. Among them, Los Angeles airport cargo accessibility exhibits a stronger increase which is mainly due to the rapid development of e-commerce, as well as the cargo passengers brought through the LA international gateway. 


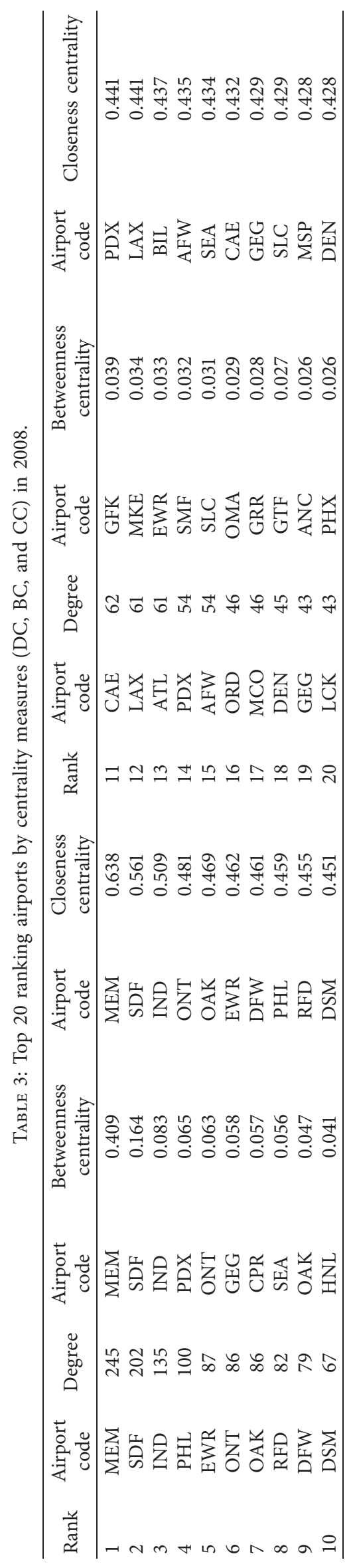




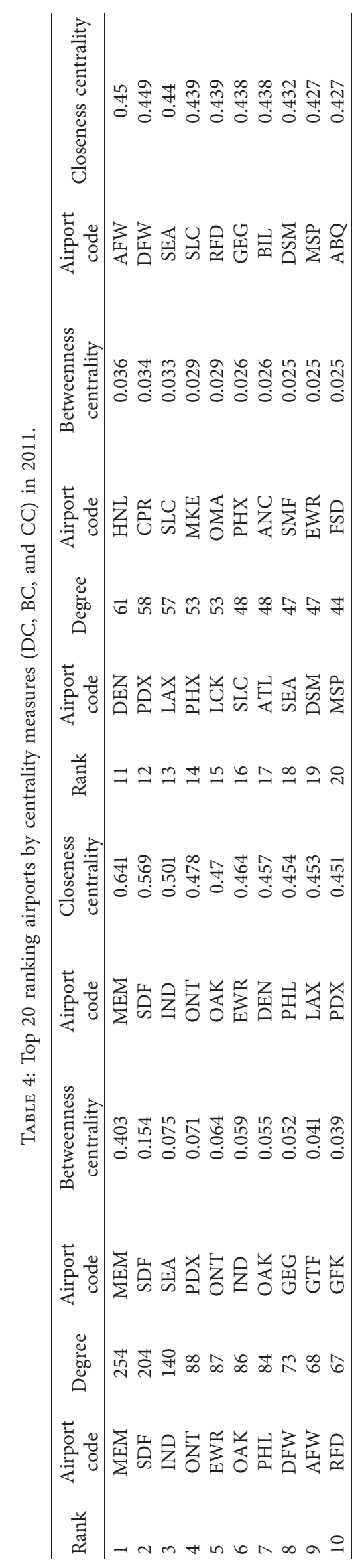




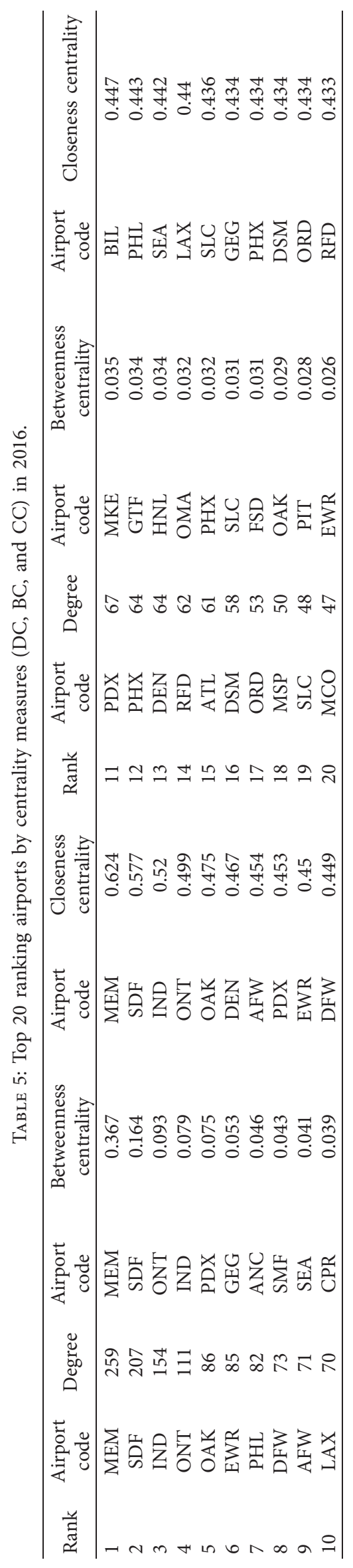




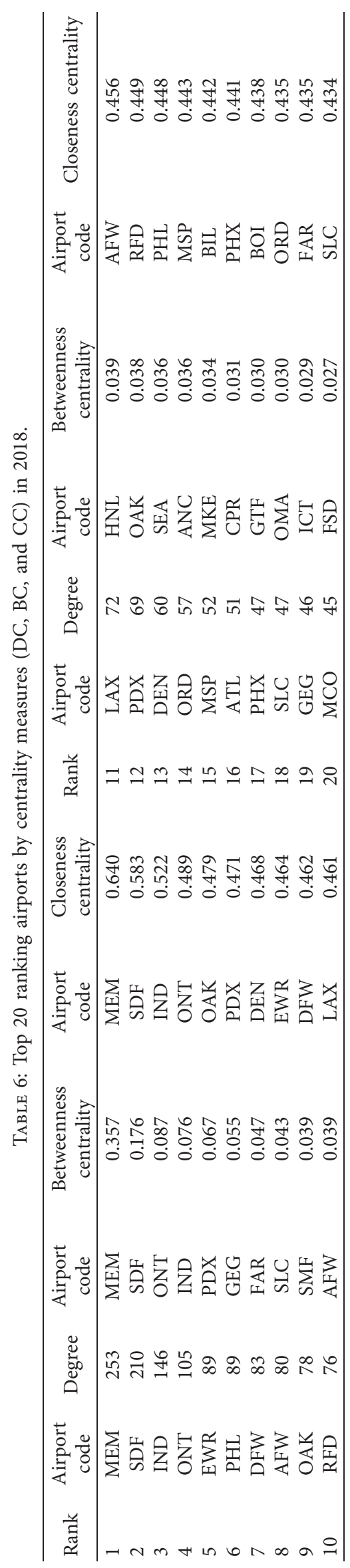




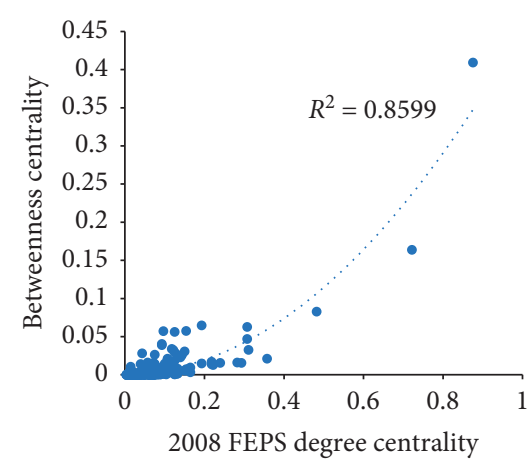

(a)

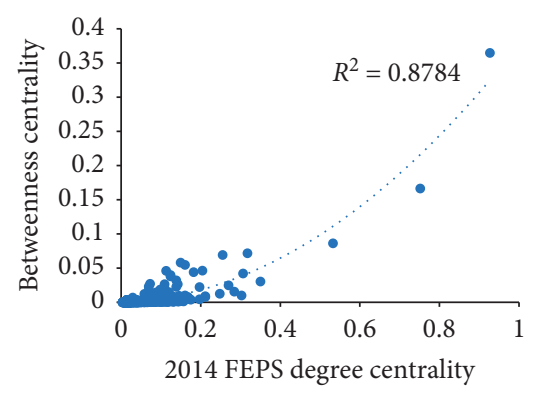

(d)

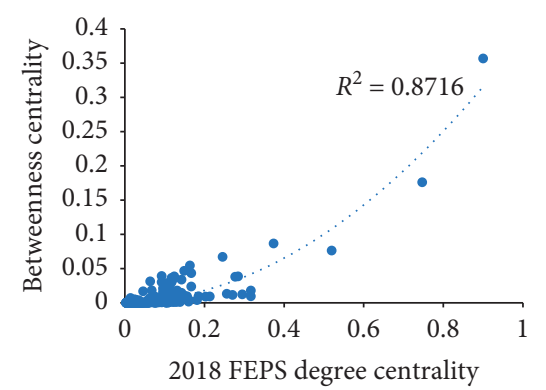

(g)

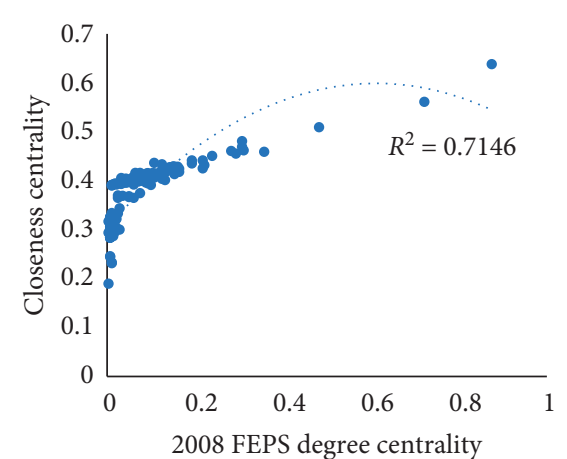

(b)

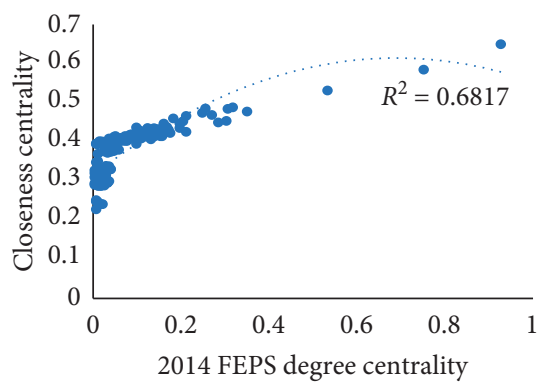

(e)

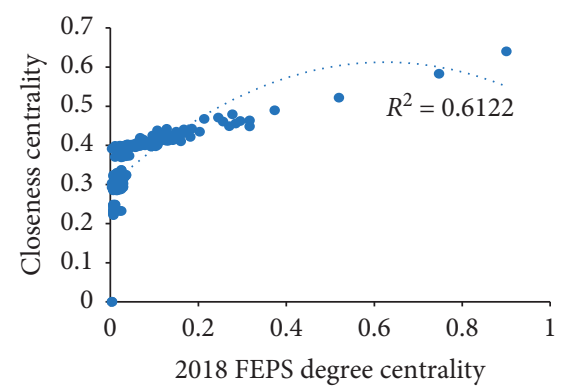

(h)

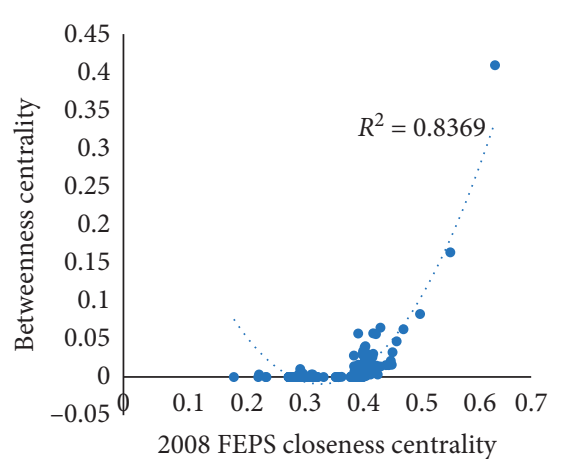

(c)

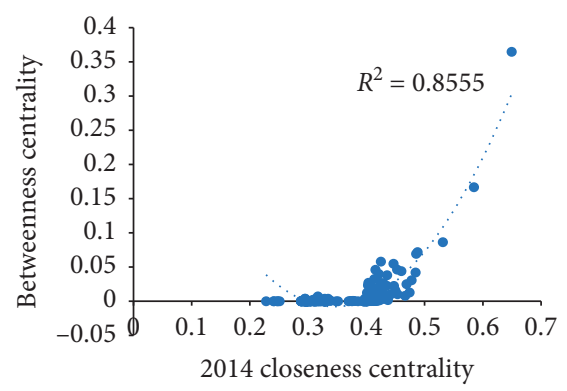

(f)

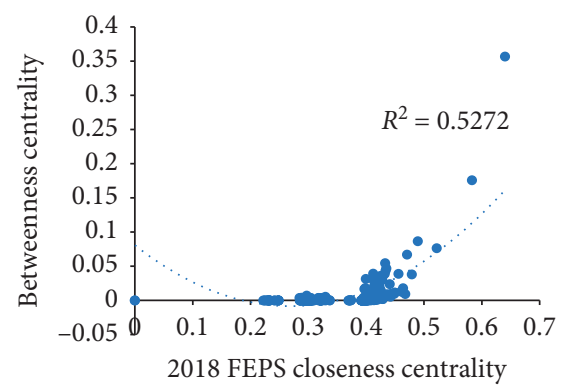

(i)

FIgURE 4: Correlation of degree centrality (DC), closeness centrality (CC), and betweenness centrality (BC) in 2008.

TABle 7: Top 20 Air Cargo Accessibility airports in the FEPS Networks (units: million ton- kilometers).

\begin{tabular}{|c|c|c|c|c|c|c|c|c|c|c|}
\hline \multirow{2}{*}{ RANK } & \multicolumn{2}{|c|}{2008 ACA } & \multicolumn{2}{|c|}{2011 ACA } & \multicolumn{2}{|c|}{2014 ACA } & \multicolumn{2}{|c|}{2016 ACA } & \multicolumn{2}{|c|}{2018 ACA } \\
\hline & Airport & FTK & Airport & FTK & Airport & FTK & Airport & FTK & Airport & FTK \\
\hline 1 & SDF & 2481.6 & SDF & 2572.2 & MEM & 2964.18 & MEM & 2843.51 & MEM & 3069.92 \\
\hline 2 & MEM & 2129.9 & MEM & 2530.93 & SDF & 2515.72 & SDF & 2598.53 & SDF & 2382.14 \\
\hline 3 & ANC & 1210.34 & ANC & 1293.82 & ANC & 1334.8 & ANC & 1553.56 & IND & 630.92 \\
\hline 4 & ONT & 515.35 & LAX & 496.65 & LAX & 611.4 & ONT & 773.22 & ONT & 675.15 \\
\hline 5 & IND & 466.25 & ONT & 483.23 & ONT & 573.58 & LAX & 743.01 & EWR & 376.95 \\
\hline 6 & OAK & 368.77 & IND & 467.79 & IND & 466.62 & IND & 611.33 & PHL & 169.78 \\
\hline 7 & LAX & 361.07 & OAK & 448.2 & OAK & 447.25 & OAK & 574.39 & DFW & 178.8 \\
\hline 8 & EWR & 318.46 & EWR & 378.58 & EWR & 364.5 & EWR & 378.57 & AFW & 69.35 \\
\hline 9 & PHL & 149.66 & SEA & 205.54 & SEA & 225.96 & PDX & 260.01 & OAK & 587 \\
\hline 10 & RFD & 144.4 & PDX & 201.56 & PDX & 202.29 & PHX & 241.28 & RFD & 132.42 \\
\hline 11 & PDX & 136.49 & PHX & 179.1 & PHX & 176.52 & SEA & 206.32 & LAX & 858.56 \\
\hline 12 & SEA & 132.3 & PHL & 126.57 & DFW & 139.25 & DFW & 171.75 & PDX & 247.1 \\
\hline 13 & SMF & 131.84 & DFW & 125.05 & PHL & 129.32 & PHL & 145.41 & DEN & 93.09 \\
\hline 14 & ORD & 125.61 & RFD & 124.96 & SLC & 104.44 & BFI & 126.95 & ORD & 71.96 \\
\hline 15 & DFW & 111.21 & SLC & 117.76 & HNL & 97.96 & SMF & 117.19 & MSP & 68.63 \\
\hline 16 & AFW & 94.88 & HNL & 96.27 & SMF & 95.74 & SLC & 115.17 & ATL & 28.46 \\
\hline 17 & SLC & 91.44 & DEN & 87.86 & RFD & 93.19 & RFD & 103.19 & PHX & 167.05 \\
\hline 18 & PHX & 85.65 & SMF & 86.58 & DEN & 91.9 & HNL & 98.32 & SLC & 142.04 \\
\hline 19 & BOS & 73.38 & BOS & 67.11 & BOS & 72.18 & $\mathrm{DEN}$ & 93.2 & GEG & 74.79 \\
\hline 20 & MIA & 68.58 & SJU & 64.27 & MIA & 66.3 & MSP & 81.17 & MCO & 53.1 \\
\hline
\end{tabular}

*Anchorage (ANC) is one of the airports in Alaska, which does not serve as a national hub in the domestic scale; but on international scale, it still serves a big role. 


\section{Conclusion and Discussion}

This paper depicts and analyzes the FEPS network from a complex network theory perspective. The major findings are summarized and discussed.

The FEPS network presents a different hub-and-spoke structure compared with its passenger counterpart, yet the air cargo network has both small-world and scale-free network characteristics since it has a small average path length and large average clustering coefficient, and its degree conforms to a power-law distribution.

Besides, the centrality indices do not comprehensively reflect the importance of nodes in the FEPS network as the accessibility measures denote that the growth in cargo volumes is not proportional to the growth of centrality value during the study period. Additionally, the correlation of node centrality shows a decreasing trend; that is, the probability of nodes in the FEPS network having high centrality values (degree, betweenness, and closeness centrality) has reduced over the years.

The core hubs in the FEPS network include MEM, SDF, IND, and ONT. The market share decreased 2\% from 2008 to 2018, partially due to FEPS restructured its aviation network after 2015, closing some nonhub airports and increasing the share of multimodal transportation. Geographically speaking, hub airports are concentrated in the Southeast, Midwest, Pacific, Northeast, and sparsely distributed in the Rocky Mountains and Southwest. Unlike air passenger networks, major hubs in the FEPS network are concentrated in small- and mid-size cities such as Memphis, Louisville, and Newark, all of which have strong capability of transshipment and cargo handling. Compared with Atlanta, Chicago, New York, and other passenger hubs, the air cargo network is separating from the passenger network. We believe that small- to mid-size cities usually have more political and economic incentives since air cargo businesses could support local and regional economic development.

The Chinese air cargo network is still in its infancy, with most of the cargo carried by passenger carriers, partially due to the development of the Chinese HSR (High-Speed-Rail). However, because of the increasing demand in international trade and domestic consumption, several private air cargo integrators have already begun taking the initiative to invest in their own dedicated air transport routes and fleets. Taking Shunfeng Express as an example, its newly built regional hub airport is located in the north part of Hubei province, which is outside the Pearl River Delta (PRD), Yangtze River Delta (YRD), Chengdu-Chongqing Economic Zone, and Bohai Sea Economic Zones, suggesting the adjustment of hub distribution and route planning, which are crucial to air cargo integrators as the market in China will continue to grow in the foreseeable future. Additionally, airport infrastructures can also bring employment opportunities, real estate development, and ultimately GDP growth. Consequently, more air transport enterprises in China have begun adjusting their network structures accordingly. Additionally, on a broader perspective, analyzing the air cargo network under different socioeconomic contexts has theoretical and application values, and therefore, future work should also take the market, corporate policy, government policy, and climate into consideration.

\section{Data Availability}

The data used to support the findings of this study are obtained from https://http://www.transtats.bts.gov/DL_SelectFields.asp? Table_ID $=292$ (US Department of Transportation Bureau of Transportation Statistics).

\section{Conflicts of Interest}

The authors declare that they have no conflicts of interest.

\section{References}

[1] IATA. https://www.iata.org/contentassets/ 4d3961c878894c8a8725278607d8ad52/air-cargo-brochure. pdf 2020.

[2] F. Wang, F. Jin, and G. Zeng, "Geographic patterns of air passenger transport in China," Scientia Geographica Sinica, vol. 23, no. 5, pp. 519-525, 2003, in Chinese.

[3] Y. Chu and Z. Gong, "The spatial pattern of China air cargo transportation in 21th century," Scientia Geographica Sinica, vol. 36, no. 3, pp. 335-341, 2016, in Chinese.

[4] Y. Chu and Z.. Yang, "Accessibility evaluation of Chinese civil airports based on airside cargo transport system," Scientia Geographica Sinica, vol. 37, no. 11, pp. 1689-1695, 2017, in Chinese.

[5] J. Jiao and J. Wang, "Spatial structure and evolution of Hainan Airlines Network: an analysis of complex network," Geographical Research, vol. 33, no. 5, pp. 926-936, 2014, in Chinese.

[6] L. Lu, Y. Wei, R. Pang et al., "Organization mode of China air passenger transport network from the perspective of aviation enterprise," Scientia Geographica Sinica, vol. 39, no. 4, pp. 550-559, 2019, in Chinese.

[7] K. Wang, Q. Gong, X. Fu, and X. Fan, "Frequency and aircraft size dynamics in a concentrated growth market: the case of the Chinese domestic market," Journal of Air Transport Management, vol. 36, no. apr, pp. 50-58, 2014.

[8] Q. Gong, K. Wang, X. Fan, X. Fu, and Y.-B. Xiao, "International trade drivers and freight network analysis-the case of the Chinese air cargo sector," Journal of Transport Geography, vol. 71, pp. 253-262, 2018.

[9] M. E. O'kelly and H. J. Miller, "The hub network design problem," Journal of Transport Geography, vol. 2, no. 1, pp. 31-40, 1994.

[10] M. E. O'kelly, "On the allocation of A subset of nodes to A mini-hub in A package delivery network," Papers in Regional Science, vol. 77, no. 1, pp. 77-99, 2005.

[11] M. E. O'kelly and Y. Lao, "Mode choice in a hub-and-spoke network: a zero-one linear programming approach," Geographical Analysis, vol. 23, no. 4, pp. 283-297, 2010.

[12] J. D. Kasarda and J. D. Green, "Air cargo as an economic development engine: a note on opportunities and constraints," Journal of Air Transport Management, vol. 11, no. 6, pp. 459-462, 2005.

[13] M. Hesse and J.-P. Rodrigue, "Global production networks and the role of logistics and transportation," Growth and Change, vol. 37, no. 4, pp. 499-509, 2006. 
[14] R. Guimera and L. A. Amaral, "Modeling the world-wide airport network," The European Physical Journal B-Condensed Matter, vol. 38, no. 2, pp. 381-385, 2004.

[15] R. Guimera, S. Mossa, A. Turtschi, and L. A. N. Amaral, "The worldwide air transportation network: anomalous centrality, community structure, and cities' global roles," Proceedings of the National Academy of Sciences, vol. 102, no. 22, pp. 7794-7799, 2005.

[16] J. T. Bowen, "A spatial analysis of FedEx and UPS: hubs, spokes, and network structure," Journal of Transport Geography, vol. 24, pp. 419-431, 2012.

[17] C. Xiu, L. Cheng, and W. Song, "Rediscovering the geographical location of Harbin based on international air cargo logistics," Geographical Research, vol. 29, no. 5, pp. 811-819, 2010, in Chinese.

[18] A. Barrat, M. Barthelemy, R. Pastor-Satorras, and A. Vespignani, "The architecture of complex weighted networks," Proceedings of the National Academy of Sciences, vol. 101, no. 11, pp. 3747-3752, 2004.

[19] A. Barrat, M. Barthélemy, and A. Vespignani, "The architecture of complex weighted networks: measurements and models," Large Scale Structure and Dynamics of Complex Networks, vol. 2, pp. 67-92, 2007.

[20] P. Malighetti, S. Paleari, and R. Redondi, "Connectivity of the European airport network: "Self-help hubbing" and business implications," Journal of Air Transport Management, vol. 14, no. 2, pp. 53-65, 2008.

[21] A. Cardillo, J. Gómez-Gardeñes, M. Zanin et al., "Emergence of network features from multiplexity," Scientific Reports, vol. 3, no. 1, 2013.

[22] J. Wang, H. Mo, F. Wang, and F. Jin, "Exploring the network structure and nodal centrality of China's air transport network: a complex network approach," Journal of Transport Geography, vol. 19, no. 4, pp. 712-721, 2011.

[23] W.-B. Du, X.-L. Zhou, O. Lordan, Z. Wang, C. Zhao, and Y.-B. Zhu, "Analysis of the Chinese airline network as multilayer networks," Transportation Research Part E: Logistics and Transportation Review, vol. 89, pp. 108-116, 2016.

[24] F. Kupfer, H. Meersman, E. Onghena, and E. Van de Voorde, "The underlying drivers and future development of air cargo," Journal of Air Transport Management, vol. 61, pp. 6-14, 2017.

[25] P. Malighetti, G. Martini, R. Redondi, and D. Scotti, "Air transport networks of global integrators in the more liberalized Asian air cargo industry," Transport Policy, vol. 80, pp. 12-23, 2019.

[26] A. Bombelli, B. F. Santos, and L. A. Tavasszy, "Analysis of the air cargo transport network using a complex network theory perspective," Transportation Research Part E Logistics and Transportation Review, vol. 138, 2020.

[27] W. G. Hansen, "How accessibility shapes land use," Journal of the American Institute of Planners, vol. 25, no. 2, pp. 73-76, 1959.

[28] P. Jia and R. Liu, "An evaluation method of airport accessibility considering land-side and air-side transport system economic geography," Economic Geography, vol. 33, no. 6, pp. 91-97, 2013.

[29] T. Verma, N. A. M. Araújo, and H. J. Herrmann, "Revealing the structure of the world airline network," Scientific Reports, vol. 4, no. 1, 2014.

[30] O. Lordan, J. M. Sallan, and P. Simo, "Study of the topology and robustness of airline route networks from the complex network approach: a survey and research agenda," Journal of Transport Geography, vol. 37, pp. 112-120, 2014.
[31] Y. Zhou, J. Wang, and G. Q. Huang, "Efficiency and robustness of weighted air transport networks," Transportation Research Part E: Logistics and Transportation Review, vol. 122, pp. 14-26, 2019.

[32] Y. Chen, J. Wang, and F. Jin, "Robustness of China's air transport network from 1975 to 2017," Physica A: Statistical Mechanics and Its Applications, vol. 539, Article ID 122876, 2020.

[33] D. J. Watts and S. H. Strogatz, "Collective dynamics of "smallworld” networks," Nature, vol. 393, no. 6684, pp. 440-442, 1998.

[34] A. Reynolds-Feighan and P. Mclay, "Accessibility and attractiveness of European airports: a simple small community perspective," Journal of Air Transport Management, vol. 12, no. 6, pp. 313-323, 2006. 